\title{
THE CATEGORY OF D-COMPLETELY REGULAR SPACES IS SIMPLE
}

BY

\author{
N. C. HELDERMANN
}

\begin{abstract}
In a recent paper $H$. Brandenburg characterized the objects of the epireflective hull of all developable spaces-that are those spaces which are homeomorphic to a subspace of a product of developable spaces-by intrinsic properties. It is shown here that these spaces, called $D$-completely regular, can be generated from a single second countable developable space $D$ which has the same cardinality as the reals. As an application of this result we obtain a new characterization of $D$-normal spaces analogous to Urysohn's lemma and a new (external) characterization of perfect spaces (meaning every closed set is a $G_{\delta}$ ).
\end{abstract}

1. Introduction. Completely regular spaces are generated by the class $\mathscr{P}$ of pseudometrizable spaces in the sense that every completely regular topology is initial with respect to all continuous mappings into objects of $\mathscr{P}$. In fact, as is obvious from the definition of a completely regular space, there is a single object in $\mathscr{P}$, namely the unit interval with its natural topology, that generates CompReg, the category of completely regular spaces.

In [2], [3] and [4] Brandenburg enlarged this framework by substituting for $\mathscr{P}$ the class $\mathscr{D}$ of developable spaces and characterized those spaces, here called $D$-completely regular spaces, which are generated by $\mathscr{D}$. In further papers [2], [5], [6], [7] he showed that a number of theorems valid in the old framework, e.g. Urysohn's and Nagata's metrization theorems, have nice counterparts in the new one. However, the question whether the analogy between these two settings would go so far as that there exists a single developable space generating all $D$-completely regular spaces remained open (see Problem 5.3 in [4]). We give here an affirmative answer by showing that it is even the unit interval that can be equipped with a developable and second countable topology that generates all $D$-completely regular spaces. As an application of this result we derive new characterizations for $D$-normal spaces, which were introduced in [2] and [5].

2. Notations and preliminaries. Topological spaces will simply be called spaces throughout this paper. Together with the continuous mappings they constitute the category Top. A subcategory $\mathcal{K}$ of Top is called epireflective, if it is closed with respect to the formation of products and subobjects. Here, all subcategories are assumed to be nonempty, full and isomorphism-closed. Every subcategory $\mathscr{K}$ is

Received by the editors August 21, 1979 and, in revised form, December 12, 1979.

1980 Mathematics Subject Classification. Primary 54D15; Secondary 54E30, 54B30, $18 B 30$.

$K e y$ words and phrases. Developable spaces, $D$-completely regular spaces, $D$-normal spaces, epireflective hull, simple category, perfect spaces. 
contained in a smallest epireflective subcategory, its epireflective hull, which we denote by $\mathrm{EH}(\mathcal{K})$. In accordance with many authors (see e.g. [9], [14], [15]) objects of $\mathrm{EH}(\mathcal{K})$ are called $\mathcal{K}$-completely regular. They are precisely those spaces that are homeomorphic to a subspace of a product of $\mathcal{K}$-objects.

A subcategory $\mathcal{K}$ of Top is called bireflective if it is closed with respect to the formation of initial sources, i.e. whenever $X$ carries the initial topology with respect to a family $\left(f_{i}: X \rightarrow K_{i}\right)_{i \in I}$ of maps into $\mathcal{K}$-objects, then $X$ belongs to $\mathcal{K}$. Every subcategory $\mathscr{K}$ of Top is contained in a smallest bireflective subcategory, its bireflective hull, which we denote by $\mathrm{BH}(\mathcal{K})$. A space $X$ belongs to $\mathrm{BH}(\mathcal{K})$ iff $X$ is initial with respect to the family of all continuous maps into $\mathcal{K}$-objects, i.e. whenever a subset $A$ of $X$ is closed in $X$ and $x \in X \backslash A$, then there exist finitely many $\mathcal{K}$-objects $K_{1}, \ldots, K_{n}$, open subsets $B_{i} \subset K_{i}$ for each $i$ and continuous functions $f_{i}: X \rightarrow K_{i}$ such that

$$
x \in f_{1}^{-1}\left[B_{1}\right] \cap \cdots \cap f_{n}^{-1}\left[B_{n}\right] \subset X \backslash A .
$$

A subcategory $\mathscr{K}$ of Top is called simple, if there exists a space $E$ such that $\mathrm{EH}(\{E\})=\mathscr{K}$.

The reader will find more details on these categorical aspects in [11]. A space $X$ is called developable [1] if there is a countable family $\left(\mathscr{Q}_{i}\right)_{i \in N}$ of open covers of $X$ such that $\left\{\operatorname{St}\left(x, Q_{i}\right) \mid i \in \mathbf{N}\right\}$ is a neighborhood base for every $x \in X$. We denote the subcategory of all developable spaces by $\mathscr{D}$. A base $\mathcal{L}$ for the closed sets of a space $X$ is called $G_{\delta}$-base, if for every $B \in \mathcal{L}$ there exists a countable family $\left(B_{i}\right)_{i \in N} \subset \mathcal{L}$ with $X \backslash B=\cup_{i \in \mathbf{N}} B_{i}$. In [3] Brandenburg characterized $D$-completely regular spaces by means of $G_{\delta}$-bases: $A$ space $X$ is $D$-completely regular iff $X$ has a $G_{\delta}$-base. The subcategory of all $D$-completely regular spaces (resp. $T_{1}$-spaces) is denoted by $\mathscr{D}$-CompReg (resp. $\mathscr{D}$-CompReg-1).

\section{The class of $D$-completely regular spaces is simple.}

3.1. We denote by $\mathbf{N}$ the positive integers, by $\mathbf{N}_{0}$ the nonnegative integers, and by $K$ the set $\{-1\} \cup \mathbf{N}_{0} \cup\left(\mathbf{N}_{0} \times \mathbf{N}\right) \cup\left(\mathbf{N}_{0} \times \mathbf{N} \times \mathbf{N}\right) \cup \ldots$ Since $K$ is countable there exists a bijection $\phi: K \rightarrow \mathbf{N}$. Define $D:=\mathbf{N}^{\mathbf{N}} \cup\{a, b\}$, where $\mathbf{N}^{\mathbf{N}}=\{f$ : $\mathbf{N} \rightarrow \mathbf{N}\}, a \neq b$ and $a, b \notin \mathbf{N}^{\mathbf{N}}$. For every $n \in \mathbf{N}$ the $n$th projection $p_{n}: \mathbf{N}^{\mathbf{N}} \rightarrow \mathbf{N}$ is defined by $p_{n}(f)=f(n)$. We consider $p_{n}^{-1}$ in a natural way as a correspondence from $\mathbf{N}$ to $D$. This allows us to define the following countable family of subsets of $D$ :

$$
\begin{gathered}
A(-1):=\{a\}, \quad A(0):=\{b\}, \\
A\left(n_{1}\right):=\{b\} \cup p_{\phi(-1)}^{-1}\left[\left\{1, \ldots, n_{1}\right\}\right] \text { for every } n_{1} \in \mathbf{N}
\end{gathered}
$$

Assume now that $A\left(n_{1}, \ldots, n_{k}\right)$ has been defined for $\left(n_{1}, \ldots, n_{k}\right) \in K \backslash\{-1\}$. We then proceed inductively by setting

$$
A\left(n_{1}, \ldots, n_{k}, n_{k+1}\right):=\left(\{a\} \cup p_{\phi\left(n_{1}, \ldots, n_{k}\right)}^{-1}\left[\left\{1, \ldots, n_{k+1}\right\}\right]\right) \backslash A\left(n_{1}, \ldots, n_{k}\right)
$$

if $k$ is odd and $n_{k+1} \in \mathbf{N}$, and

$$
A\left(n_{1}, \ldots, n_{k}, n_{k+1}\right):=\left(\{b\} \cup p_{\phi\left(n_{1}, \ldots, n_{k}\right)}^{-1}\left[\left\{1, \ldots, n_{k+1}\right\}\right]\right) \backslash A\left(n_{1}, \ldots, n_{k}\right)
$$

if $k$ is even and $n_{k+1} \in \mathbf{N}$. 
Let $\mathscr{D}^{\prime}$ denote the family of all $A\left(n_{1}, \ldots, n_{k}\right)$ obtained in this way and $\mathscr{D}$ all finite unions of elements of $\mathscr{D}$ '. Clearly, $\mathscr{D}$ is a base for the closed sets of a topology on $D$. The space thus obtained is denoted $\mathbf{D}$.

3.2. THEOREM. $\mathbf{D}$ is a second countable developable space.

Proof. It is well known from [5] that every $D$-completely regular second countable space is developable. Since $\mathscr{D}$ is countable it suffices to show that every element of $\mathscr{D}$ is a $G_{\delta}$-set. But this follows easily from the fact that every $A\left(n_{1}, \ldots, n_{k}\right)$ is a $G_{\delta}$-set and finite unions of $G_{\delta}$-sets are again $G_{\delta}$-sets.

We will henceforth make no notational difference between the space $\mathbf{D}$ and the underlying set of $\mathbf{D}$.

3.3. Remarks. (1) To provide more insight into the structure of $\mathbf{D}$ we give here an explicit construction for a development of $\mathbf{D}$ :

For $n \in \mathbf{N}_{0}$ define $\mathcal{Q}(n):=\{\mathbf{D} \backslash A(n), \mathbf{D} \backslash A(-1)\}$ and for $\left(n_{1}, \ldots, n_{k}\right) \in K$, $k>1$, we set

$$
\mathcal{Q}\left(n_{1}, \ldots, n_{k}\right):=\left\{\mathbf{D} \backslash A\left(n_{1}, \ldots, n_{k}\right), \mathbf{D} \backslash A\left(n_{1}, \ldots, n_{k-1}\right)\right\} .
$$

Let $\Psi: \mathbf{N} \rightarrow K \backslash\{-1\}$ be a bijection. We then define $\mathcal{L}(1):=\mathbb{Q}(\Psi(1))$, and if, for $n \in \mathbf{N}, \mathcal{L}(n)$ is given, we set

$$
\mathcal{L}(n+1):=\mathscr{L}(n) \wedge \mathfrak{Q}(\Psi(n+1))
$$

where $\wedge$ denotes elementwise intersection. It is easily verified that the countable family $\beta=\{\mathcal{L}(n) \mid n \in \mathbf{N}\}$ is a development for $\mathbf{D}$.

(2) $\mathbf{D}$ is an $R_{0}$-space ${ }^{1}$ since it is developable. However, $\mathbf{D}$ is not $T_{0}$ : Define $f$ : $\mathbf{N} \rightarrow \mathbf{N}$ and $g: \mathbf{N} \rightarrow \mathbf{N}$ by

$$
f(n):=\left\{\begin{array}{ll}
2 & \text { if } n=\phi(-1), \\
1 & \text { otherwise, }
\end{array} \quad \text { and } \quad g(n):= \begin{cases}2 & \text { if } n=\phi(-1) \\
2 & \text { if } n=\phi(2) \\
1 & \text { otherwise. }\end{cases}\right.
$$

Then the only difference between $f$ and $g$ is their value in the $\phi(2)$ th component. Let us assume that there exists an open set $B$ such that $f \notin B$ and $g \in B$. We may assume that

$$
B=\mathbf{D} \backslash \cup\left\{A(k) \mid k \in K^{\prime}, K^{\prime} \subset K \text { finite }\right\} .
$$

This implies $f \in A(k)$ and $g \notin A(k)$ for some $k \in K$. But the only $A(k)$ 's cutting into the $\phi(2)$ th component are of the form $A(2, n)$ for $n \in \mathbf{N}$. Hence, there is $n \in \mathbf{N}$ with $f \in A(2, n)$ and $g \notin A(2, n)$. Since $\mathbf{D} \backslash A(2)=\cup_{n \in N} A(2, n)$ this implies $f \notin A(2)$-a contradiction because $f \in p_{\phi(-1)}^{-1}(2) \subset A(2)$. The same argumentation is still valid if the roles of $f$ and $g$ are interchanged.

3.4. Lemma. Let $X$ be a space, and $A, B$ two disjoint closed sets with the property that there is a countable family $\mathcal{L}$ of closed sets satisfying the following conditions:

(1) $\{A, B\} \subset \mathcal{L}$,

(2) for every $B \in \mathcal{L}$ there is a family $(B(n))_{n \in N} \subset \mathcal{L}$ with

\footnotetext{
${ }^{1}$ A topological space $(X, \mathrm{cl})$ is called $R_{0}$-space or symmetric if $x \in \operatorname{cl}\{y\}$ implies $y \in \operatorname{cl}\{x\}$ for all $x$, $y \in X$.
} 


$$
X \backslash B=\bigcup\{B(n) \mid n \in \mathbf{N}\},
$$

(3) $\mathcal{L}$ is closed with respect to finite unions.

Then there exists a continuous mapping $f: X \rightarrow \mathbf{D}$ such that $f^{-1}(a)=A$ and $f^{-1}(b)=$ $B$.

Proof. For every $\left(n_{1}, \ldots, n_{k}\right) \in K=\{-1\} \cup \mathbf{N}_{0} \cup\left(\mathbf{N}_{0} \times \mathbf{N}\right) \cup\left(\mathbf{N}_{0} \times \mathbf{N} \times \mathbf{N}\right)$ $\cup \cdots$ we define a set $C\left(n_{1}, \ldots, n_{k}\right) \in \mathcal{L}$ by induction. Define $C(-1):=A$ and $C(0):=B$. According to condition (2) there exists a family $(B(n))_{n \in N} \subset \mathcal{L}$ with $X \backslash A=\cup\{B(n) \mid n \in \mathbf{N}\}$. Define for $n \in \mathbf{N} C(n):=B \cup \cup\{B(l) \mid l \leqslant n\}$. We assume that $C\left(n_{1}, \ldots, n_{k}\right) \in \mathcal{L}$ is given for $\left(n_{1}, \ldots, n_{k}\right) \in K \backslash\{-1\}$. Then there exists a family $(D(n))_{n \in N} \subset \mathcal{L}$ such that

$$
X \backslash C\left(n_{1}, \ldots, n_{k}\right)=\cup\{D(n) \mid n \in \mathbf{N}\} .
$$

Define, in case $k$ is odd,

$$
C\left(n_{1}, \ldots, n_{k}, n_{k+1}\right):=A \cup \cup\left\{D(l) \mid l \leqslant n_{k+1}\right\},
$$

and, if $k$ is even,

$$
C\left(n_{1}, \ldots, n_{k}, n_{k+1}\right):=B \cup \cup\left\{D(l) \mid l \leqslant n_{k+1}\right\} .
$$

For $x \in X$ let $K(x):=\left\{\left(n_{1}, \ldots, n_{k}\right) \in K \mid x \in C\left(n_{1}, \ldots, n_{k}\right)\right\}$. Recall that for every $k \in K$ a set $A(k) \subset \mathbf{D}$ was defined in 3.1. We assert now, that for every $x \in X$ the set

$$
S(x):=\bigcap\{A(k) \mid k \in K(x)\}
$$

is nonempty.

Let $x \in X$ be arbitrarily given. If $x \in A$, then we clearly obtain $S(x)=\{a\}$, and similarly $S(x)=\{b\}$ if $x \in B$. We assume $x \in X \backslash(A \cup B)$. Define

$$
K_{\min }(x):=\left\{\left(n_{1}, \ldots, n_{k}\right) \in K(x) \mid n_{k}=1 \text { or } x \notin C\left(n_{1}, \ldots, n_{k-1}\right)\right\} .
$$

Note that $C\left(n_{1}, \ldots, n_{k}\right) \subset C\left(n_{1}, \ldots, \bar{n}_{k}\right)$ whenever $n_{k} \leqslant \bar{n}_{k}$. If $\left(n_{1}, \ldots, n_{k-1}, n_{k}\right)$ $\in K_{\min }(x)$ and $\left(n_{1}, \ldots, n_{k-1}, \bar{n}_{k}\right) \in K_{\min }(x)$, this implies $n_{k}=\bar{n}_{k}$. Therefore, the mapping $g: \mathbf{N} \rightarrow \mathbf{N}$, defined below, is well defined:

$$
g(i):= \begin{cases}n_{1}, & \text { if } i=\phi(-1), \text { where } n_{1} \text { denotes the unique element of } K_{\min }(x) \\ & \text { having just one component, } \\ n_{k}, & \text { if } i=\phi\left(n_{1}, \ldots, n_{k-1}\right) \text { and }\left(n_{1}, \ldots, n_{k}\right) \in K_{\min }(x) \text { and } k>1, \\ 1, & \text { otherwise. }\end{cases}
$$

We prove by induction

$$
g \in A\left(n_{1}, \ldots, n_{k}\right) \Leftrightarrow\left(n_{1}, \ldots, n_{k}\right) \in K(x) .
$$

Assume $k=1$. Then $\left(n_{1}\right) \in K(x)$ implies $x \in C\left(n_{1}\right)$; hence $g(\phi(-1))<n_{1}$ and we obtain $g \in A\left(n_{1}\right)$. Conversely, $g \in A\left(n_{1}\right)$ implies $g(\phi(-1))<n_{1}$, and we conclude $\left(n_{1}\right) \in K(x)$.

Assume $k>1$ and the assertion to be true for all $l<k$. If $\left(n_{1}, \ldots, n_{k}\right) \in$ $K(x)$, then $x \in C\left(n_{1}, \ldots, n_{k}\right) \subset X \backslash C\left(n_{1}, \ldots, n_{k-1}\right)$. Hence, $x \notin$ $C\left(n_{1}, \ldots, n_{k-1}\right)$ which implies $\left(n_{1}, \ldots, n_{k-1}\right) \notin K(x)$. Using the assertion for $k-1$ we obtain $g \notin A\left(n_{1}, \ldots, n_{k-1}\right)$. Since $g\left(\phi\left(n_{1}, \ldots, n_{k-1}\right)\right) \leqslant n_{k}$ this implies 
$g \in A\left(n_{1}, \ldots, n_{k}\right)$. Conversely, we assume $g \in A\left(n_{1}, \ldots, n_{k}\right)$ which yields $g\left(\phi\left(n_{1}, \ldots, n_{k-1}\right)\right) \leqslant n_{k}$ and $g \notin A\left(n_{1}, \ldots, n_{k-1}\right)$. Therefore, $\left(n_{1}, \ldots, n_{k-1}\right) \notin$ $K(x)$ which implies $x \notin C\left(n_{1}, \ldots, n_{k-1}\right)$. Since $X \backslash C\left(n_{1}, \ldots, n_{k-1}\right)=$ $\cup_{l \in \mathbf{N}} C\left(n_{1}, \ldots, n_{k-1}, l\right)$ there exists $l \in \mathbf{N}$ with $x \in C\left(n_{1}, \ldots, n_{k-1}, l\right)$. Hence

$$
g\left(\phi\left(n_{1}, \ldots, n_{k-1}\right)\right)=\min \left\{l \in \mathbf{N} \mid x \in C\left(n_{1}, \ldots, n_{k-1}, l\right)\right\}
$$

which yields $x \in C\left(n_{1}, \ldots, n_{k}\right)$, i.e. $\left(n_{1}, \ldots, n_{k}\right) \in K(x)$. It is now obvious that $S(x) \neq \varnothing$ since $g \in S(x)$.

We are now prepared to define a mapping $f: X \rightarrow \mathbf{D}$ by assigning to every $x \in X$ an arbitrary element $f(x)$ from $S(x)$. To show that $f$ is continuous it is evidently sufficient to show that for every $\left(n_{1}, \ldots, n_{k}\right) \in K, f^{-1}\left[A\left(n_{1}, \ldots, n_{k}\right)\right]$ is closed in $X$. Therefore, let $\left(n_{1}, \ldots, n_{k}\right) \in K$ be given. We show that

$$
f^{-1}\left[A\left(n_{1}, \ldots, n_{k}\right)\right]=C\left(n_{1}, \ldots, n_{k}\right),
$$

which implies immediately that $f$ is continous since all $C$ 's are closed. Let $x \in C\left(n_{1}, \ldots, n_{k}\right)$ be given. Then

$$
f(x) \in S(x)=\cap\left\{A\left(\bar{n}_{1}, \ldots, \bar{n}_{l}\right) \mid x \in C\left(\bar{n}_{1}, \ldots, \bar{n}_{l}\right)\right\} \subset A\left(n_{1}, \ldots, n_{k}\right) ;
$$

hence $x \in f^{-1}\left[A\left(n_{1}, \ldots, n_{k}\right)\right]$. Conversely assume $x \in f^{-1}\left[A\left(n_{1}, \ldots, n_{k}\right)\right]$, i.e. $f(x)$ $\in A\left(n_{1}, \ldots, n_{k}\right)$, and, moreover, $x \notin C\left(n_{1}, \ldots, n_{k}\right)$. Then there exists $i \in \mathbf{N}$ such that $x \in C\left(n_{1}, \ldots, n_{k}, i\right)$ in case $n_{1} \geqslant 0$, or $x \in C(i)$ in case $n_{1}=-1$. This implies, as shown above, $f(x) \in A\left(n_{1}, \ldots, n_{k}, i\right)$ in case $n_{1} \geqslant 0$ and $f(x) \in A(i)$ in case $n_{1}=-1$, but in any case a contradiction to $f(x) \in A\left(n_{1}, \ldots, n_{k}\right)$ since $A\left(n_{1}, \ldots, n_{k}\right) \cap A\left(n_{1}, \ldots, n_{k}, i\right)=\varnothing(A(-1) \cap A(i)=\varnothing$, respectively). The last argumentation yields, in particular, $f^{-1}(a)=f^{-1}[A(-1)]=C(-1)=A$, and similarly $f^{-1}(b)=B$.

3.5. THEOREM. The class of D-completely regular spaces is simply generated by $\mathbf{D}$, i.e. $\operatorname{EH}(\{\mathbf{D}\})=\mathscr{D}$-CompReg.

Proof. Since $\mathbf{D}$ is not $T_{0}$, the epireflective hull and the bireflective hull of $\mathbf{D}$ coincide [12], [13]. We show that, for an arbitrary $D$-completely regular space $X$ and for $x \in X \backslash D$, where $D$ is closed in $X$, there exists a continuous mapping $f$ : $X \rightarrow \mathbf{D}$ and an open set $V$ in $\mathbf{D}$ such that $x \in f^{-1}[V] \subset X \backslash D$.

Choose a $G_{\delta}$-base $\mathcal{L}^{\prime}$ for the closed sets of $X$. Then $\mathcal{L}:=\wedge \vee \mathcal{L}^{\prime}$ is a $G_{\delta}$-base which is closed with respect to finite unions, where $\wedge \vee \mathcal{L}^{\prime}$ denotes the family of all finite intersections of finite unions of elements of $\mathcal{L}^{\prime}[2,3.1 .17]$. There exists $A \in \mathcal{L}$ such that $x \in X \backslash A \subset X \backslash D$, and, since there is a family $(B(n))_{n \in \mathbb{N}} \subset \mathcal{L}$ with $\cup\{B(n) \mid n \in \mathbf{N}\}=X \backslash A, B \in \mathcal{L}$, such that $x \in B$ and $A \cap B=\varnothing$. Clearly, the conditions of Lemma 3.4 are fulfilled for $A, B$ and $\mathcal{L}$. We conclude therefore, that a continuous map $f: X \rightarrow \mathbf{D}$ exists, fulfilling $f^{-1}(a)=A$ and $f^{-1}(b)=B$. Since $V:=\mathbf{D} \backslash\{a\}$ is open, we obtain $x \in f^{-1}[V]=X \backslash A \subset X \backslash D$.

To show the converse assume that $X$ is initial with respect to the family of all continuous maps to $\mathbf{D}$. We constructed in 3.1 a family $\mathscr{D}$ of subsets of $\mathbf{D}$ and we remarked in 3.2 that $\mathscr{D}$ is a $G_{\delta}$-base for the closed sets of $D$. Define $\mathscr{B}^{\prime}:=\left\{f^{-1}[A] \mid A \in \mathscr{D}, f: X \rightarrow \mathbf{D}\right.$ continuous $\}$ and $\mathscr{B}:=\wedge \mathscr{B}^{\prime}$. We show that 
$\mathscr{B}$ is a $G_{\delta}$-base for $X$ : Clearly, $\mathscr{B}$ is a base. Let $B \in \mathscr{B}$ be given. Then $B=$ $\cap{ }_{i=1}^{n} C_{i}$ and $C_{i}=\cup_{j \in M_{i}} D_{i j}$ for all $i \in\{1, \ldots, n\}$, where $M_{i}=\left\{1, \ldots, m_{i}\right\} \subset$ $\mathbf{N}$ and $D_{i j}=f_{i j}^{-1}\left[A_{i j}\right]$ for some continuous functions $f_{i j}: X \rightarrow \mathbf{D}$ and $A_{i j} \in \mathscr{D}$. An easy computation yields

$$
B=\bigcup\left\{D_{1 j_{1}} \cap \cdots \cap D_{n j_{n}} \mid\left(j_{1}, \ldots, j_{n}\right) \in M_{1} \times \cdots \times M_{n}\right\} .
$$

This implies

$$
X \backslash B=\bigcap\left\{\left(X \backslash D_{1 j_{1}}\right) \cup \cdots \cup\left(X \backslash D_{n j_{n}}\right) \mid\left(j_{1}, \ldots, j_{n}\right) \in M_{1} \times \cdots \times M_{n}\right\} .
$$

Since there exists for every $i \in\{1, \ldots, n\}$ and every $j \in M_{i}$ a countable family $(E(i, j, k))_{k \in \mathbf{N}} \subset \mathscr{D}$ such that $\mathbf{D} \backslash A_{i j}=\cup_{k \in \mathbf{N}} E(i, j, k)$ we can write

$$
\begin{aligned}
X \backslash B=\bigcap\left\{\left(\bigcup_{k \in \mathbf{N}} f_{1 j_{1}}^{-1}\left[E\left(1, j_{1}, k\right)\right]\right) \cup \cdots\right. & \cup\left(\bigcup_{k \in \mathbf{N}} f_{n j_{n}}^{-1}\left[E\left(n, j_{n}, k\right)\right]\right) \mid \\
& \left.\left(j_{1}, \ldots, j_{n}\right) \in M_{1} \times \cdots \times M_{n}\right\} .
\end{aligned}
$$

Now define $L:=M_{1} \times \cdots \times M_{n}$ and for $l=\left(j_{1}, \ldots, j_{n}\right) \in L$

$$
F(l, k):=f_{1 j_{1}}^{-1}\left[E\left(1, j_{1}, k\right)\right] \cup \cdots \cup f_{n j_{n}}^{-1}\left[E\left(n, j_{n}, k\right)\right] .
$$

Note that $F(l, k) \in \bigvee \mathscr{B}^{\prime}$. The above expression can now be reformulated as

$$
X \backslash B=\bigcap\left\{\bigcup_{k \in \mathbf{N}} F(l, k) \mid l \in L\right\}=\bigcup_{g \in N^{L}} \bigcap_{l \in L} F(l, g(l)) .
$$

Since $L$ is finite we obtain $\bigcap_{l \in L} F(l, g(l)) \in \mathscr{B}$ for every $g \in \mathbf{N}^{L}$. Hence, $X \backslash B$ is expressed above as a countable union of elements of $\mathscr{B}$. This proves that $\mathscr{B}$ is a $G_{\delta}$-base and that $X$ is $D$-completely regular.

4. The class of $D$-completely regular $T_{1}$-spaces is simple. For an $R_{0}$-space $X$ and $x, y \in X$ we define $x \sim y$ iff $\operatorname{cl}\{x\}=\operatorname{cl}\{y\}$. Then $\sim$ is an equivalence relation and the set $\omega X$ of equivalence classes equipped with the final topology with respect to the natural mapping $\omega: X \rightarrow \omega X$ is usually called the $T_{1}$-reflection of $X$. Since D is developable, $\omega \mathbf{D}$, which we denote henceforth by $\mathbf{D}_{1}$, is a developable second countable $T_{1}$-space [16].

4.1. THEOREM. The class of D-completely regular $T_{1}$-spaces is simply generated by
D $_{1}$.

Proof. Since $\mathbf{D}$ is initial with respect to $\omega$ and $\mathbf{D}_{1}$, we have $\operatorname{BH}\left(\left\{\mathbf{D}_{1}\right\}\right)=\mathscr{D}$ CompReg. From [12, 3.4.2(1)] and [13, Theorem 3] it can easily be derived that for every class $\mathscr{K}$ of $T_{1}$-spaces $\operatorname{EH}(\mathscr{K})=\mathrm{BH}(\mathcal{K}) \cap\left\{T_{1}\right.$-spaces $\}$ always holds. This implies the assertion.

4.2. Proposition. $D_{1}$ has cardinality c.

Proof. $D_{1}$ has obviously cardinality less than or equal to $c$. We prove the assertion by mapping $\{1,2\}^{\mathbf{N}}$ into $\mathbf{D}$ by a map $x$ such that $\omega \circ x$ is an injection.

Let $f \in\{1,2\}^{\mathbf{N}}$ be given. Then $L:=\left\{s_{i} \mid i \in \mathbf{N}_{0}\right\}$, where $s_{0}=-1, s_{1}=f(1), \ldots$, $s_{i}=(f(1), \ldots, f(i))$, is a subset of $K$, which was defined in $\S 3$. Define 
$x(f):=\left(n_{1}, n_{2}, \ldots\right) \in \mathbf{N}^{\mathbf{N}}$, where

$$
n_{\phi(k)}= \begin{cases}f(i+1)+1, & \text { if } k=s_{i} \text { for some } i \in \mathbf{N}_{0}, \\ 1, & \text { otherwise. }\end{cases}
$$

Then $x(f) \in A(f(1)+1) \backslash A(f(1))$ holds and one shows by induction for all $n \in \mathbf{N}, n>1$,

$$
x(f) \in A(f(1), f(2), \ldots, f(n-1), f(n)+1) \backslash A(f(1), \ldots, f(n)) .
$$

Let $f, g \in\{1,2\}^{\mathbf{N}}$ be given with $f \neq g$. Define

$$
n:=\min \{i \in \mathbf{N} \mid f(i) \neq g(i)\} .
$$

We assume for simplicity that $f(n)=1$ and $g(n)=2$. From the argumentation above we obtain $x(f) \in A(f(1), \ldots, f(n-1), 2)$ and

$$
x(g) \in A(f(1), \ldots, f(n-1), 3) \backslash A(f(1), \ldots, f(n-1), 2) .
$$

Since $A(f(1), \ldots, f(n-1), 2)$ is closed, this implies $\operatorname{cl}\{x(f)\} \neq \operatorname{cl}\{x(g)\}$ and hence $\omega \circ x$ is an injection.

In consequence of Proposition 4.2 the unit interval $[0,1]$ can be equipped with a topology such that the resulting space $I$ is homeomorphic to $\mathbf{D}_{1}$, and that $a$ corresponds to 0 and $b$ corresponds to 1 . Hence $I$ is a developable and second countable $T_{1}$-space.

4.3. Corollary. A topological space $X$ is $D$-completely regular iff for all $x \in X$ and closed sets $A \subset X$ with $x \in X \backslash A$ there exists a continuous function $f: X \rightarrow I$ such that $f(x)=0$ and $f(y)=1$ for all $y \in A$.

Lemma 3.4 and the construction of $\mathbf{D}_{1}$ above have an immediate consequence for perfect spaces. Recall that a space is called perfect if every closed set is a $G_{\delta}$. Clearly, every developable space is perfect.

4.4. THEOREM. The following conditions are equivalent for a space $X$.

(1) $X$ is perfect,

(2) for every pair of disjoint closed subsets $A$ and $B$ of $X$ there exists a continuous function $f: X \rightarrow \mathbf{D}_{1}$ and distinct points $a$ and $b$ in $\mathbf{D}_{1}$ such that $f^{-1}(a)=A$ and $f^{-1}(b)=B$,

(3) for every closed subset $A$ of $X$ there exists a continuous function $f: X \rightarrow \mathbf{D}_{1}$ and $a \in \mathbf{D}_{1}$ such that $f^{-1}(a)=A$.

In [7] a class $\varepsilon$ of spaces was called adequate for functional separation, if for every space $X$ and every pair of disjoint $\delta$-closed subsets $A$ and $B$ of $X$ there exists an $\mathcal{E}$-object $E$, two distinct points $a$ and $b$ in $E$, and a continuous function $f$ : $X \rightarrow E$ such that $\operatorname{cl} f[A] \subset\{a\}$ and $\operatorname{cl} f[B] \subset\{b\}$. It remained open in the above-mentioned paper, whether the class of developable spaces is adequate for functional separation.

4.5. Proposition. The class $\mathcal{D}$ of developable spaces is adequate for functional separation.

Proof. Let $\mathscr{D}$-closed disjoint subsets $A$ and $B$ of a space $X$ be given. Then there exist developable spaces $Y_{1}$ and $Y_{2}$, closed subsets $C_{1}$ of $Y_{1}$ and $C_{2}$ of $Y_{2}$, and 
continuous functions $f_{1}: X \rightarrow Y_{1}$ and $f_{2}: X \rightarrow Y_{2}$ with $f_{1}^{-1}\left[C_{1}\right]=A$ and $f_{2}^{-1}\left[C_{2}\right]=$ $B$. The initial topology $\mathcal{X}$ on $X$ with respect to $f_{1}$ and $f_{2}$ is developable, and it is easily verified that $\operatorname{cl}_{\mathfrak{X}} A \cap \operatorname{cl}_{\mathfrak{X}} B=\varnothing$. The assertion follows now immediately from Theorem 4.4.

5. $D$-normal spaces. Normal space can be viewed as those spaces $X$ with the property that for every pair of disjoint closed sets $A$ and $B$ in $X$ there exists a pseudometrizable space $Y$ and a continuous mapping $f: X \rightarrow Y$ such that $\operatorname{cl} f[A] \cap$ $\operatorname{cl} f[B]=\varnothing$. In analogy Brandenburg $[2,3.1 .24]$, defined a space $X$ to be $D$-normal, if for every pair of disjoint closed sets $A$ and $B$ there exists a developable space $Y$ and a continuous mapping $f: X \rightarrow Y$ such that $\operatorname{cl} f[A] \cap \operatorname{cl} f[B]=\varnothing$.

The following result provides characterizations of $D$-normal spaces analogous to the separation of disjoint closed sets by disjoint open sets and Urysohn's lemma for normal spaces.

5.1. THEOREM. For a space $X$ the following conditions are equivalent.

(1) $X$ is $D$-normal,

(2) for every pair of disjoint closed sets $A$ and $B$ there exist disjoint closed $G_{\delta}$-sets $C$ and $D$ such that $A \subset C$ and $B \subset D$,

(3) whenever $A$ is closed and contained in an open set $U$, there exists an open $F_{\sigma}$-set $F$ with $A \subset F \subset U$,

(4) whenever $A$ is closed and contained in an open set $U$, there exists a closed $G_{\delta}$-set $G$ with $A \subset G \subset U$,

(5) for every pair of disjoint closed sets $A$ and $B$ there exists a continuous mapping $h: X \rightarrow I$ such that $h(x)=0$ whenever $x \in A$ and $h(x)=1$ whenever $x \in B$.

Proof. (1) $\Rightarrow(2)$. Let disjoint closed sets $A$ and $B$ be given. Then there exists a developable space $Y$ and a continuous map $f: X \rightarrow Y$ such that $\operatorname{cl} f[A] \cap \operatorname{cl} f[B]=$ $\varnothing$. Since developable spaces are perfect, $\operatorname{cl} f[A]$ and $\operatorname{cl} f[B]$ are closed $G_{\delta}$-sets in $Y$. This implies that $C:=f^{-1}[\operatorname{cl} f[A]]$ and $D:=f^{-1}[\operatorname{cl} f[B]]$ are $G_{\delta}$-sets in $X$ with the desired properties.

(2) $\Rightarrow(3) \Rightarrow(4)$ is clear. (4) $\Rightarrow(5)$. Let disjoint closed sets $A$ and $B$ be given. We define for every

$$
\left(n_{1}, \ldots, n_{k}\right) \in K^{\prime}=\mathbf{N}_{0} \cup(\mathbf{N} \times \mathbf{N}) \cup(\mathbf{N} \times \mathbf{N} \times \mathbf{N}) \cup \ldots
$$

a closed $G_{\delta}$-set $G\left(n_{1}, \ldots, n_{k}\right)$. From (4) we obtain a closed $G_{\delta}$-set $A(0)$ with $A \subset A_{0} \subset X \backslash B$. Since $A(0)$ is $G_{\delta}$ there is a family $(F(n))_{n \in \mathrm{N}}$ of closed sets such that $A(0)=\cap\{X \backslash F(n) \mid n \in \mathbf{N}\}$, and for each $n \in \mathbf{N}$ there exists a closed $G_{\delta}$-set $A(n)$ with $F(n) \subset A(n) \subset X \backslash A(0)$. Now let the closed $G_{\delta}$-set $A\left(n_{1}, \ldots, n_{k}\right)$ be defined. Then there exists a family $(H(n))_{n \in \mathrm{N}}$ of closed sets such that

$$
A\left(n_{1}, \ldots, n_{k}\right)=\cap\{X \backslash H(n) \mid n \in \mathbf{N}\},
$$

and for every $n \in \mathrm{N}$ there exists a closed $G_{\delta}$-set $A\left(n_{1}, \ldots, n_{k}, n\right)$ such that

$$
H(n) \subset A\left(n_{1}, \ldots, n_{k}, n\right) \subset X \backslash A\left(n_{1}, \ldots, n_{k}\right) \text {. }
$$

We define

$$
\mathcal{L}(A):=\left\{A\left(n_{1}, \ldots, n_{k}\right) \mid\left(n_{1}, \ldots, n_{k}\right) \in K^{\prime}\right\}
$$


and by proceeding analogously for $B$ we obtain

$$
\mathcal{L}(B)=\left\{B\left(n_{1}, \ldots, n_{k}\right) \mid\left(n_{1}, \ldots, n_{k}\right) \in K^{\prime}\right\},
$$

starting with a closed $G_{\delta}$-set $B(0)$ which satisfies $B \subset B(0) \subset X \backslash A(0) \subset X \backslash A$.

Let $\mathcal{L}$ denote the set of all finite intersections of finite unions of elements of $\mathcal{L}(A) \cup \mathcal{L}(B)$. The property of $\mathcal{L}(A) \cup \mathcal{L}(B)$ that for every $D \in \mathcal{L}(A) \cup \mathcal{L}(B)$ there exists a family $(D(i))_{i \in \mathrm{N}} \subset \mathcal{L}(A) \cup \mathcal{L}(B)$ such that

$$
X \backslash D=\bigcup\{D(i) \mid i \in \mathbf{N}\}
$$

is still valid for $\mathcal{E}$. The proof is identical to that of 3.1.17 in [2]. Thus $\mathcal{E}$ fulfills all conditions of Lemma 3.4 which yields a continuous function $f: X \rightarrow \mathbf{D}$ with $f^{-1}(a)=A(0)$ and $f^{-1}(b)=B(0)$. Combining the natural mapping $\omega: \mathbf{D} \rightarrow \mathbf{D}_{1}$ with the homeomorphism between $D_{1}$ and $I$ we obtain a continuous function $g: D \rightarrow I$ with $g(a)=0$ and $g(b)=1$. Hence $h:=g \circ f: X \rightarrow I$ has the desired properties $h[A] \subset\{0\}$ and $h\{B\} \subset\{1\} .(5) \Rightarrow(1)$ is trivial. Thus, the proof is complete.

The following statement is obvious from Theorem 5.1.

\subsection{Corollary. Every perfect space is D-normal.}

The results obtained here and in [2], [3], [5] and [6] encourage further research on the relationships between developable, $D$-normal and $D$-completely regular spaces viewed as being "symmetric" to pseudometrizable, normal and completely regular spaces. A promising extension of this new framework is motivated by condition (3) of Theorem 5.1: Call a space $X D$-regular if every point $x \in X$ has a neighborhood base consisting of open $F_{\sigma}$-sets. In addition to the obvious implications it is already known from [5] that every $D$-regular Lindelöf space is $D$-normal. Further results on $D$-regular spaces are contained in a forthcoming paper of the author [10].

6. Acknowledgements and remarks. During the preparation of this paper the author was informed that A. Mysior from Gdansk University, Poland, proved independently the simplicity of $\mathscr{D}$-CompReg and $\mathscr{D}$-CompReg-1. His universal space, however, has very high cardinality and is of weight $2^{c}$. We would like to express our thanks to $\mathrm{H}$. Brandenburg who carefully read the first draft of this paper. Further results on $D$-normal spaces will appear in a paper of his, which is in preparation [8].

ADDED IN PROOF. The results of A. Mysior mentioned above have been published in the meantime (Two remarks on D-regular spaces, Glasnik Mat. 15 (35) (1980), 153-156).

\section{REFERENCES}

1. R. H. Bing, Metrization of developable spaces, Canad. J. Math 3 (1951), 175-186.

2. H. Brandenburg, Hüllenbildungen für die Klasse der entwickelbaren topologischen Räume, Dissertation, Freie Universität, Berlin, 1979.

3. __ On a class of nearness spaces and the epireflective hull of developable topological spaces, Proc. Topology Conf., Belgrad, 1977 (to appear).

4. __ Die epireflektive Hülle der entwickelbaren topologischen Räume, Nordwestdeutsches Kategorienseminar (Bericht der Tagung in Bielefeld, 1977), G. Richter, Ed., Bielefeld, 1978, pp. 33-49.

5. , On spaces with a $G_{\delta}$-basis, Arch. Math. (Basel) (to appear). 
6. ___ Some characterizations of developable spaces, Proc. Amer. Math. Soc. 80 (1980), 157-161.

7. On E-normal spaces (Proc. Conf. Categorical Topology, Berlin, 1978), H. Herrlich and G.

Preuss, Eds., Lecture Notes in Math., vol. 719, Springer-Verlag, Berlin and New York, 1979, pp. $24-34$.

8. Separating closed sets by continuous mappings into developable spaces, in preparation.

9. R. Engelking and S. Mrowka, On E-compact spaces, Bull. Acad. Polon. Sci. Sér. Sci. Math. Astronom. Phys. 6 (1958), 429-436.

10. N. C. Heldermann, Developability and some new regularity axioms, Canad. J. Math. (to appear).

11. H. Herrlich and G. E. Strecker, Category theory: an introduction, 2nd ed., Sigma Series in Pure Math., Vol. 1, Heldermann Verlag, Berlin, 1979.

12. T. Marny, Rechts-Bikategoriestrukturen in topolologischen Kategorien, Dissertation, Freie Universität, Berlin, 1973.

13. __ On epireflective subcategories of topologiccal categories, General Topology Appl. 10 (1979), 175-181.

14. S. Mrowka, Further results on E-compact spaces. I, Acta Math. 120 (1968), 161-185.

15. A. Mysior, On generalized classes of complete regularity, Bull. Acad. Polon. Sci. Sér. Sci. Math. Astronom. Phys. 24 (1976), 341-342.

16. J. M. Worrell, Jr., Upper semicontinuous decompositions of developable spaces, Proc. Amer. Math. Soc. 16 (1965), 485-490.

Zentralblatt für Mathematik, Otto-Suhr-Allee 26-28, D-1000 Berunn 10, Federal Republic OF Germany 